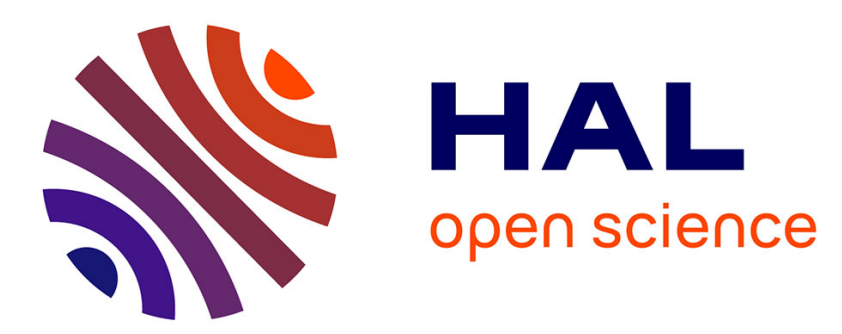

\title{
THEORY OF ELECTRON-PHONON INTERACTION AND BROKEN SYMMETRIES OF SEMICONDUCTOR INTERFACES
}

\author{
W. Hanke, A. Muramatsu
}

\section{- To cite this version:}

W. Hanke, A. Muramatsu. THEORY OF ELECTRON-PHONON INTERACTION AND BROKEN SYMMETRIES OF SEMICONDUCTOR INTERFACES. Journal de Physique Colloques, 1984, 45 (C5), pp.C5-385-C5-394. 10.1051/jphyscol:1984559 . jpa-00224178

HAL Id: jpa-00224178

https://hal.science/jpa-00224178

Submitted on 1 Jan 1984

HAL is a multi-disciplinary open access archive for the deposit and dissemination of scientific research documents, whether they are published or not. The documents may come from teaching and research institutions in France or abroad, or from public or private research centers.
L'archive ouverte pluridisciplinaire HAL, est destinée au dépôt et à la diffusion de documents scientifiques de niveau recherche, publiés ou non, émanant des établissements d'enseignement et de recherche français ou étrangers, des laboratoires publics ou privés. 


\title{
THEORY OF ELECTRON-PHONON INTERACTION AND BROKEN SYMMETRIES OF SEMICONDUCTOR INTERFACES
}

\author{
W. Hanke and A. Muramatsu*
}

\begin{abstract}
Max-Planek-Institut fur Festkörperforschung, Heisenbergstrasse 1, D-7000 Stuttgart 80, E.R.G.

*Institute for Theoretical Physics, University of California, Santa Barbara, Cal. 93106, U.S.A.
\end{abstract}

\begin{abstract}
Résumé - Nous présentons brièvement les résultats de nos travaux récents sur la théorie des excitations collectives et leurs liaisons avec les instabilités structurales sur les surfaces des semiconducteurs. La discussion est basée sur un traitement en fonction de Green des excitations électroniques et électron-phonons, avec une attention spéciale au cas des surfaces où une approximation de "jel1ium" ne convient pas. En abordant les effets à $N$ corps par une approximation de Hartree-Fock écrantée dëpendant du temps, nous obtenons les conditions pour 1'apparition d'une instabilité électronique (densité de charge ou de spin) à la surface et le couplage de 1'onde de densité de charge résultante au réseau. Des calculs quantitatifs pour un film de huit plans (111) de Si conduit à une instabilité de la surface paramagnétique idéale par rapport à l'apparition d'andes de densitê de spin avec une longueur d'onde correspondant aux surstructures ( $2 \times 1$ ) et ( $7 \times 7$ ) qui sont observées. Comparées au volume, nous trouvons que les interactions à plusieurs particules (en particulier l'interaction électrontrou dans l'état de triplet) dans la fonction de Green à deux particules, augmentent de façon significative en surface, c'est-à-dire lorsque 1 'inhomogénéité de la charge augmente. Un calcul de phonons pour la surface Si(11) montre que le couplage de l'onde de densité de charge en réseau conduit à un mode de surface mou. Nos études des modes électroniques et de phonons expliquent le point de départ de 1'instabilité mais ne donne pas la configuration du nouvel êtat fondamental. Cependant la description des excitations par la fonction de Green peut aussi être un outil pour vérifier les déterminations de 1 'état de base des diagrammes de reconstruction vis-à-vis des résultats expérimentaux.
\end{abstract}

Abstract - A summary is presented of recent investigations we have performed on the theory of collective excitations and their interrelation with structural instabilities on semiconductor surfaces. The discussion is based on a Green's function treatment of electronic and electronphonon excitations, with special emphasis on surfaces for which the jellium approximation does not hold. Treating many-body effects within time-dependent screened Hartree-Fock (TDSHF), we extract the conditions for the appearance of an electronic (charge- and spin-density) instability at the surface and the coupling of the resulting charge-density wave to the lattice. Quantitative calculations for an eight-layer Si(111) slab display an instability of the ideal paramagnetic surface with respect to spin-density waves, with wavelength corresponding to the observed $(2 \times 1)$ and $(7 \times 7)$ superstructures. Compared with the bulk, we find the many-many particle (in particular the triplet-state electron-hole) interactions in the two-particle Green's function to become significantly enhanced at the surface, i.e., where the charge inhomogeneity increases. A calculation of the phonons for the Si(111) surface demonstrates that the CDW coupling to the lattice leads to a soft sur- 
face mode. Our studies of electronic and phonon modes explain what drives the instability initially and not what is the new ground-state configuration. However, we also elaborate on the Green's function excitational description as a tool to check ground-state determinations of reconstruction patterns against experiment.

\section{Introduction}

The field of elementary excitations of electronic and of electron-phonon nature on surfaces has recently gained strong impetus from a variety of experimental techniques, such as photoemission, low-energy electron diffraction (LEED) ${ }^{2}$, characteristic energy-loss ${ }^{3}$ and scattering of light (He) atoms ${ }^{4}$. For bulk solids, the theory of the corresponding response and correlation functions, which serve as a unifying perturbation concept of elementary excitations and the many-body aspects, is in good accord with a large variety of experiments ${ }^{5}-7$.

This review gives a summarizing account of recent work ${ }^{8-}{ }^{10}$ which aims (i) at a similar microscopic description of surface elementary excitations where one starts, like in the complementary bulk studies from the general Green's function many-body theory and, (ii) a quantitative understanding of spin- and charge-density-wave (SDW and CDW) instabilities at semiconductor surfaces.

Previous descriptions in semiconductors and insulators ${ }^{11}$ of the surface response functions, and thus also of the extracted elementary excitations, rest usually on phenomenological models using often macroscopic boundary conditions. Successful examples are the polariton theory 11,12 and models for surface phonons ${ }^{11}$, where the force constants are taken as parameters, usually adjusted to bulk values. These models were and are quite valuable in clarifying for example symmetry aspects of surface phonons ${ }^{12}$. However, in contrast to the models our aim here is to derive the force constants from first principles, in particular connecting them with the underlying electronic structure.

In sec. II we summarize our treatment of the two-particle Green's function. A local orbital representation allows for an explicit solution of the Bethe Salpeter equation within TDSHF. It takes into account manybody effects of random phase (RPA) and electron-hole (excitonic) nature as well as the microscopic charge-density profile in the surface region. The resulting spin and density response functions present a practical scheme for a microscopic calculation of surface elementary excitations in conducting as well as non-conducting solids.

With help of this formalism we study in sec. III the conditions for the appearance of an electronic (charge- and spin-density) instability at the surface and the coupling of the resulting charge-density wave (CDW) to the lattice.

Quantitative calculations of the spin- and density-response function of the ideal Si(111) surface are reviewed in secs. III and IV. They display pronounced RPA and excitonic effects and lead to spin-density instability for wavevectors corresponding to the observed $(2 \times 1)$ and $(7 \times 7)$ reconstructions. An a-priori calculation of the phonons of an 8-1ayer slab of Si(111) shows that the coupling of the CDW's to the lattice leads to a soft surface mode with atomic displacements corresponding to ionic "buckling". Implications of these findings for other surface systems 14,15 and general aspects of the importance of many-body effects for the a-priori determination of surface structures are discussed. 


\section{Bethe-Salpeter Equation and the Elementary Excitation Spectrum}

Backbone of our theoretical description is an explicit expression for the two-particle Green's functior, obtained in a local-orbital description of one-electron states in a finite slab. It allows for a microscopic derivation of ground-state and excitational properties embedded in the surface charge- and spin-density response functions. A detailed discussion of this loca-orbital formalism has been given elsewhere ${ }^{58}$. Here, we only introduce the notations and results needed in the following discourse.

The two-particle propagator satisfies the Bethe-Salpeter equation ${ }^{5}$

$$
\begin{aligned}
G\left(11^{\prime} ; 22^{\prime}\right) & =G^{\circ}\left(11^{\prime} ; 22^{\prime}\right)+ \\
& +G^{\circ}\left(11^{\prime} ; 33^{\prime}\right) \text { I }\left(33^{\prime} ; 44^{\prime}\right) G\left(44^{\prime} ; 22^{\prime}\right)
\end{aligned}
$$

where I is the irreducible electron (e) - hole (h) interaction within the time-dependent screened Hartree-Fock (TDSHF) approximation. Integrations over repeated indices are understood. Despite its formidably complicated integral-equation structure, one can derive a directly accessible expression for the solution of eq. (1). To this we introduce a Iocal representation for the surface Bloch wave functions and use the formalism of Hanke and Sham ${ }^{5}$

$\phi_{n} \vec{k}(\vec{r})=\frac{1}{\sqrt{N M}} \sum_{, \gamma} c_{n, \gamma}^{m}(\vec{k}) \sum_{\ell} e^{i \vec{k} \cdot \vec{k}} a_{\gamma}^{m}\left(\vec{r}-\vec{k}_{\ell}\right)$

Here $\vec{k}$ is the $2-D$ wavevector in the $m$-th layer in the slab with $M$ layers, $\vec{R}$ a 2-D translation vector and $R_{m}$ a $3-D$ basis vector for the $2-D$ unit cêl1. $\gamma$ gives the orbital index and $N$ the number of $2-D$ unit cells.

Inserting eq. (2) in eq. (1), a matrix equation follows, which can be inverted to extract the two-particle propagator $G$ (in matrix notation)

$\mathrm{G}(\overrightarrow{\mathrm{q}} ; \omega)=\mathrm{G}^{\mathrm{O}}(\overrightarrow{\mathrm{q}} ; \omega)\left(1+\mathrm{G}^{\mathrm{O}}(\overrightarrow{\mathrm{q}} ; \omega)\left(\mathrm{V}(\overrightarrow{\mathrm{q}} ; \omega)-\frac{1}{2} V^{\mathrm{S}}(\overrightarrow{\mathrm{q}}, \omega)\right)\right)^{-1}$

The non-interacting e-h propagator is given by

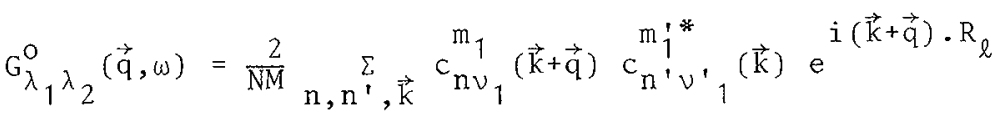

$$
\begin{aligned}
& x \frac{f_{n}(\vec{k}+\vec{q})-f_{n^{\prime}}(\vec{k})}{\omega+E_{n}(\vec{k})-E_{n}(\vec{k}+\vec{q})} e^{-i(\vec{k}+\vec{q}) \cdot R_{\ell}} \frac{m}{2}_{c_{n v_{2}}}(\vec{k}+\vec{q}) c_{n^{\prime} v^{\prime}}(\vec{k})
\end{aligned}
$$

$E_{n}(\vec{k})$ and $f(\vec{k})$ are energies and occupation numbers of the slab Bloch states. The $n_{\text {RPA }}$ (or time-dependnet Hartree) Coulomb matrix with

$$
\begin{array}{r}
V_{\lambda \lambda^{\prime}}^{c}(\vec{q})=\sum_{s} e^{i \vec{q} \cdot \vec{R}_{s}} \iint d^{3} r^{\prime} d^{3} r a_{\mu}^{*}\left(\vec{r}-\vec{R}_{\ell}-\vec{k}_{s}\right) x \\
x \quad a_{\nu}\left(\vec{r}-\vec{k}_{s}\right) v\left(\vec{r}-\vec{r}^{\prime}\right) a_{\nu^{*}}^{*}\left(\vec{r}^{\prime}\right) a_{\mu},\left(\vec{r}^{\prime}-\vec{R}_{\ell^{\prime}}\right)
\end{array}
$$

is the exchange interaction to the electron-hole attraction ${ }^{5}$. It gives 
the local-field effects, a fact that can be seen from Eq. (5) where $V^{c}$ is expressed as the summation of the interactions of a "dipole" (Electron-hole pair) at the lattice site $\mathrm{R}=0$ with "dipoles" at lattice sites $\vec{R}=\vec{R}_{S}$, which are induced through the motion of a collective excitation along the system. $V^{S}$ is on the other hand the screened electron-hole attraction:

$$
\begin{aligned}
& V_{\lambda \lambda^{\prime}}^{S}(\vec{q})=\sum_{s} e^{i \vec{q} \cdot \vec{R}_{S}} \iiint d^{3} r d^{3} r^{\prime} d^{3} r^{\prime \prime} a_{\mu}^{*}\left(\vec{r}-\vec{R}_{\ell^{-}} \vec{R}_{m}\right) \quad x \\
& x a_{\nu}\left(\vec{r}^{\prime}-\vec{R}_{m}\right) v\left(\vec{r}-\vec{r}^{\prime \prime}\right) \varepsilon^{-1}\left(\vec{r}^{\prime \prime}, \vec{r}^{\prime}\right) a_{\nu^{\prime}}^{*}\left(\vec{r}^{\prime}\right) a_{\mu},\left(\vec{r}-\vec{R}_{\ell^{\prime}}\right)
\end{aligned}
$$

Using eqs. (3) to (6), we can extract an explicit expression for the nonlocal dielectric function of the surface. In Fourier space $(\vec{G}, \vec{G} \cdot$ are reciprocal lattice vectors), we write

$$
\begin{aligned}
\varepsilon^{-1}\left(\overrightarrow{\mathrm{q}}+\overrightarrow{\mathrm{G}}, \overrightarrow{\mathrm{q}}+\overrightarrow{\mathrm{G}}^{\prime} ; z, z^{\prime}\right)=\delta_{G G}, \delta\left(z-z^{\prime}\right) & +\int v\left(|\overrightarrow{\mathrm{q}}+\overrightarrow{\mathrm{G}}|,\left|z-z^{\prime \prime}\right|\right) . \\
\cdot & x\left(\overrightarrow{\mathrm{q}}+\overrightarrow{\mathrm{g}}, \overrightarrow{\mathrm{q}}+\overrightarrow{\mathrm{G}}^{\prime} ; z^{\prime \prime}, z^{\prime}\right) d z^{\prime \prime},
\end{aligned}
$$

where $v(|\vec{q}|,|z|)$ is the 2-D Fourier transform of the bare Coulomb potential, and $x$ the density-response function

$x=\sum_{\lambda, \lambda}, A_{\lambda}(\vec{q}+\vec{G} ; z)\left(G_{0}^{-1}+\left(V^{C}-\frac{1}{2} V^{S}\right)\right)^{-1} A_{\lambda}^{*},\left(\vec{q}+\overrightarrow{G^{\prime}}, z^{\prime}\right)$

$A_{\lambda}$ are the charge-density form factors

$A_{\lambda}(\vec{q}+\vec{G} ; z)=\int d^{2} r a_{\nu}^{*}\left(\vec{r} ; z-R_{m}^{z}\right) e^{-i(\vec{q}+\vec{G}) \cdot \vec{r}{ }^{\prime}} a_{v^{\prime}}^{m^{\prime}}\left(\vec{r}-\vec{R} \lambda_{\lambda} ; z-R_{m}^{z}\right)$

III. Magnetic and Charge-Density Instabilities: Semiconductor Surfaces.

On the basis of the above theory for two-particle excitations, we can give explicit conditions for an electronic instability.

It will appear whenever the system has a tendency to show charge-density fluctuations, that is if collective excitations can arise without having delivered any energy to the system. This happens when the following resonance condition is fulfilled in eq. (6) for $\omega=0$ :

$\operatorname{det}\left|\mathrm{G}^{\mathrm{O}^{-1}}(\overrightarrow{\mathrm{q}} ; \omega=0)+\mathrm{V}_{\mathrm{XC}}(q)\right| \equiv 0$

where $V_{x C}(\vec{q})=V^{C}(\vec{q})-\frac{1}{2} V(\vec{q})$.

This condition corresponds to an instability of the system against formation of a charge-density wave of wavelength $2 \pi / \vec{q}$. A similar condition is obtained for a spin-density wave instability:

$$
\left|G^{O^{-1}}(\vec{q}, \omega=0)-\frac{1}{2} V^{S}(\vec{q})\right|=0
$$


where only triplet states of the electron-hole pairs are considered ${ }^{16}$. In this case $V^{c}$ does not contribute because this interaction takes place only for singlet states.

Similar conditions to (10) and (11), which are exact in the screened Hartree-Fock approximation, were derived in an approximated way by Fedders and Martin ${ }^{15}$ and Chan and Heine ${ }^{17}$.

The condition (10) shows that the appearance of an electronic instability depends on the balance of effects due to nesting of the Fermi surface, this information being contained in $\mathrm{G}^{\circ}(\vec{q})$, the local-field effects $\left(V^{C}\right)$ and the excitonic effects. In particular, a tendency to a metal-insulator transition which is due to a special geometry of the Fermi surface will be suppressed by strong local-field effects which could appear in a system with very localized electrons. On the other hand the electron-hole attraction can favour such an instability producing a situation where $V^{X}{ }^{x}$. Moreover, as can be seen in Eq. (11), a system that shows a tendency to a CDW instability in the TDSHF approximation will have an even stronger tendency to a SDW instability in the same approximation ${ }^{18}$.

A detailed numerical study of these many-body effects on the ideal Si(111) surface has been given in refs. 9,10. This study, which was done for an eight-layer slab, was based on an LCAO representation of a self-consistent pseudopotential bandstructure. All aspects of the numerical surface approach have been tested also in bulk. In particular, the calculated bulk properties (optical response and quasi-particle bands $)^{5,6}$ based on the same elementary excitation approach are in quite good accord with experiment.

Fig. 1 displays the results we have found for the electronic instabilities on the ideal $\$$ if 111$)$ surface in three different approximations (plotted in (detG) , $d=$ matrix dimension=113), The dotted line gives the Hartree approximation $\left(V_{x c}=0\right)$ and thus contains only the bandstructure effects. The system is obviously stable, and small Fermisurface nesting effects due to the dangling-bond (DB) metallic band,

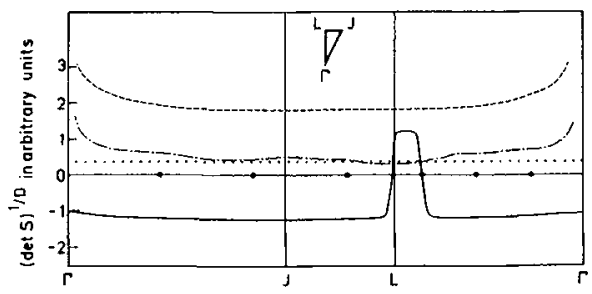

Fig. 1. SDW instabilities on the ideal Si(111) surface. For an explanation see the text. The inset shows the irreducible part of the 2D Brillouin zone. The dots on the abscissa correspond to wave vectors for the possible $(7 \times 7)$ superstructures along the $\Gamma-J-L-\Gamma$ direction.

are washed out by the interaction with the substrate and in the substrate itself. The dashed ine shows the RPA results $\left(V^{C} \neq 0\right)$. Here the $(1 \times 1)$ structure is stabilized even further. A ( $1 / q)$ divergence is observed at the $\Gamma$ point due to the metali ic $D B$ band. If we additionally take the screened $e-h$ attraction $\left(-\frac{1}{2} V^{s}\right)$ into account in the dask-dotted line, the determinant goes essentially back to the Hartree results, with a small dip near l point. This dip suggests a tendency towards a chargedensity wave j.n that region. However, an actual instability can only appear when simultaneously the coupling to the ions is considered. As discussed in the next section, then a phonon instability around the Lpoint, appears corresponding to the $(2 \times 1)$ superstructure.

So much for possible instabilities towards charge-density fluctuations. The spin-density (SDW) fluctuations are given in Fig. 1 by the full 
line. Here the determinant has indeed zeros at two wave vectors corresponding to the observed $(2 \times 1)$ (L-point) and $(7 \times 7)$ reconstructions. The role of SDW fluctuations for the $(2 \times 1)$ has also been pointed out in local (spin-) density groundstate calculation, employing the pseudopotential scheme ${ }^{19}$. On the basis of our results it would be interesting to search for magnetic surface superstructures for example, by means of spin-polarized photoemission experiments.

However, we have to stress one point here: our sole aim was to investigate what drives an instability initially, (for example, in the Si(111) configuration) and not what is the new groundstate configuration.

Recently, for the $(2 \times 1)$-reconstructed surface in Si(111), electronic surface state dispersion relations from angle- resolved photoemission data20 were compared with theoretical results obtained from a localdensity (LDA) single-particle band-structure 22. Though the LDA eigenvalues are formally not justified to be taken as single-electron excitations, the unfavorable comparison was taken to imply that the bucklingtype geometrical structure is not compatible with experiment. Spindensity (LSD) functional calculations ${ }^{9}$, as well as calculations modelling intra-site Coulomb correlation a la Hubbard ${ }^{3}$, yield two occupied surface bands consistent with one of the experimental photoemission data $^{20}$. More recent photoemission results, on the other hand, find only one occupied surface-state band ${ }^{21}$. A novel -bonded chain structural model was then proposed by Pandey 2 and, on the bas is of LDA eigenvalues, found to be in good accord with the photoemission data. Selfconsistent LDA total-energy calculations yielded for the -bonded chain model the, so far, lowest energy and - in contrast to previous calculations-a raising for the total energy for buckling-type of reconstructions ${ }^{22}$.

In view of the very small (of order $0.05 \mathrm{eV}$ ) energy differences per atom on the surface of different proposed reconstruction models, it seems important to have an alternative and independent access to surface instabilities. This is provided by the Green's function formalism which can be used, starting from a proposed equilibrium configuration (for example, the $\pi$-bonded chain), to check whether its elementary excitation spectrum is in accordance with experiment. This crucial check is presentiy undertaken for the $\pi$-bonded chain model.

The next section gives a short summary of a microscopic calculation of phonon energies for an ideal $\mathrm{Si}(111) \mathrm{slab}^{8}, 10$. On the one hand, to the best of our knowledge this is the first phonon calculation, which is parameterfree and has as the only input the bandstructure energy, wave functions and ionic (pseudo-)potential. On the other hand, this calculation serves as an example for another elementary excitation from which we can gain insight into what drives the instability initially on the ideal surface.

\section{Microscopic Theory of Surface Lattice Dynamics and Application to Si(111).}

With the help of the density-response function of sec. II, we can now discuss the surface lattice dynamics of a slab configuration with surfaces perpendicular to the $z$-direction. ${ }^{8}, 10$

The dynamical matrix contains an electronic and a bare ionic part. The latter can be constructed by standard means, and is given elsewhere ${ }^{3}$. The electronic contribution, due to the renormalization of the ion-ion interaction by the many-electron system, can be expressed in the harmonic approximation with the help of the density-response function ${ }^{5}$. 
By Fourier-transforming the ionlc potential $W$ and the expression $(8,9)$ for the susceptibility, we can obtain the electronic contribution to the dynamical matrix ${ }^{8}$.

$\mathrm{D}_{\alpha \beta}^{\mathrm{E}}\left(\kappa \kappa^{\prime}, \vec{q}\right)=\overline{\mathrm{D}}_{\alpha \beta}^{\mathrm{E}}\left(\kappa \kappa^{\prime}, \vec{q}\right)-\overline{\mathrm{D}}_{\alpha \beta}^{\mathrm{E}}\left(\kappa^{\prime}, 0\right)$

with

$$
\begin{aligned}
& \overline{\mathrm{D}}_{\alpha \beta}^{\mathrm{E}}\left(\kappa \kappa^{\prime}, \overrightarrow{\mathrm{q}}\right)=\left(\mathrm{M}_{\alpha} \mathrm{M}_{\alpha}\right)^{-1 / 2} \mathrm{x} \\
& \times \sum_{\lambda \lambda}, F_{\alpha}^{\lambda}(\kappa, \vec{q}) \mathrm{S}_{\lambda \lambda},(\vec{q}) \mathrm{F}_{\beta}^{\lambda^{\prime}+}\left(\kappa^{\prime}, \vec{q}\right)
\end{aligned}
$$

where

$$
\begin{aligned}
& F_{\alpha}^{\lambda}(\kappa, \vec{q})=i \sum_{\vec{G}}(\vec{q}+\vec{G})_{\alpha} e^{i \vec{G} \cdot \vec{r}_{\kappa}^{0} / /} x \\
& x \int d z W\left(\vec{q}+\vec{G},,\left|z-\vec{R}_{\kappa z}^{0}\right|\right) \cdot A_{\lambda}(\vec{q}-\vec{b}, z)
\end{aligned}
$$

for $\alpha=x, y$ and

$$
F_{z}^{\lambda}(k, \vec{q})=-\sum_{G}|\vec{q} \cdot \vec{r}| e^{i \vec{G} \cdot \vec{R}_{k}^{O} / /} x
$$

$$
x \int d z \operatorname{sgn}\left(z-R_{K z}^{0}\right) W\left(\vec{q}+\vec{G},\left|z-R_{K z}^{0}\right|\right) \cdot A_{\lambda}\left(\vec{q}+\vec{G}^{\prime}, z\right)
$$

The force form factor $\mathrm{F}_{\alpha}^{\lambda}$ correspond to a force in direction $\alpha$ on the ion $k$ at the site $\vec{R}_{K}^{\alpha}$ due to interaction with a charge-density wave $A_{\lambda}$. We can see from (13) that in the case of an electronic instability given by (10) a strong negative correction from the electronic contribution will appear and produce a phonon instability.

This is born out in an explicit calculation we have performed for Si(111), employing the same two-particle screening propagator, as discussed in the last section.

We obtain two kinds of surface modes in Fig. 2: low-energy modes and high-energy modes. We identify the first ones as Rayleigh modes ${ }^{4,25}$ because they are elliptically polarized in the sagittal plane and appear below the bulk-like modes. The high-energy modes are transyerse modes, polarized parallel to the surface. They were also obtained by zimmer$\operatorname{mann}^{2}{ }^{6}$ and Ludwig ${ }^{2}$ in the same range of frequencies with a phenomenological model for a semi-infinite si crystal.

Two types of instabilities affect the Rayleigh modes in our calculation: in the region where $a \lesssim 1 / l$, $\ell$ being the thickness of the $s 1 a b$, we obtain phonon instabilities which are related to the fact that we are dealing with an unrelaxed surface (violation of infinitesimal rotational invariance), and also that we did not implement the acoustic sum rule for the surface ${ }^{8}$, its application being presently carried out. 
The instabilities of the Rayleigh modes at the zone boundary are due to the coupling of the CDW, discussed in Section III, with the lattice. An analysis of the eigenvectors of these modes, which are elliptically polarized in the sagittal plane and strongly localized in the outmost layer, shows that the displacement of the surface atoms give rise to a "buckled" surface.

In order to obtain a better understanding of the mechanism which produces a softening of the surface modes at $q_{B}$, we concentrate on the top layer of the slab and take into account $\mathrm{BZ}$ 'ny the dangling-bond

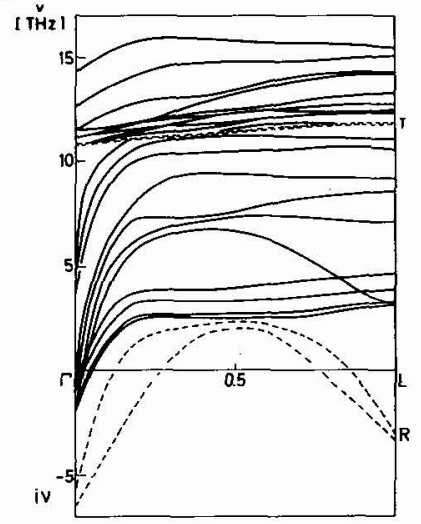

Fig.2. Phonon spectrum for $q=(0, \xi)$ for an ideal 8-1ayer slab of $\mathrm{Si}(111)$. Dashed $1 \mathrm{i}-$ nes: surface modes.

(DB) orbitals. In Fig. 3 we show the contribution to the susceptibility from the non-interacting electron-hole system. The band structure gives rise to maxima near to wavevectors that correspond to the $7 \times 7$ reconstruction due to "nesting" of the Fermi surface ${ }^{28}$. But no structure corresponding to the $2 \times 1$ superstructure is observable. The RPA local-field effects give a large correction (Fig. 3) due to the extremely localized nature of the DBornitals. The features present before disappear and no electronic instability is observable. Beyond RPA, the system shows a strong tendency to an excitonic insulator instability when electron-hole attraction is considered. In this approximation the excitation energy of the system is lowered giving an enhancement of density response (Fig.3) and a tendency of the DB's to form a CDW. We can distinguish two features conected with commonly observed superstructures: First, we obtain a saddle-point at L. This enhancement of the susceptibility is responsible for the surface phonon instabilities at the $\mathrm{L}$ point (Fig.2). But being a saddle point, the

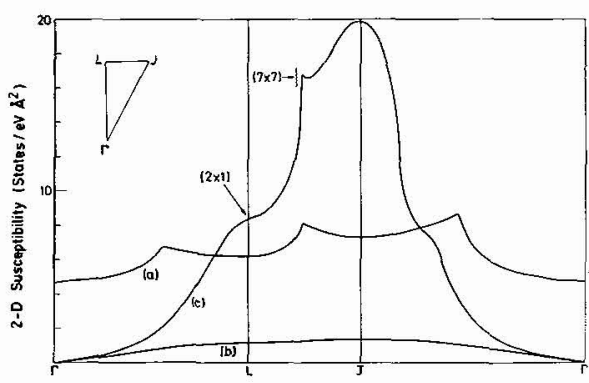

Fig. 3. 2-D susceptibility for a) non-interacting el-hole system; b) plus local field effects and c) plus e1.-hole attraction. The inset shows the irreducible part of the Brillouin zone. fact indicates that the CDW can almost only couple with displacements perpendicular to the surface as corroborated in the full calculation. This "buckling" displacement patshould be still unstable against other configurations. In fact we observe that the next local maximum appears for a wavevector nearly corresponding to the $(7 \times 7)$ reconstruction. Another maximum appears at $J$, corresponding to a $(\sqrt{3} \times \sqrt{3}) 30^{\circ}$ superstructure, which is occasionally observed 29 .

The coupling of the CDW with the lattice, given by the form factor $F^{s}(k, \vec{q})$ determines the type of reconstruction. We observe that the coupling factor of the $\mathrm{DB}-\mathrm{CDW}$ to displacements parallel to the surface is zero at the zone boundary. The on $1 y$ contribution for displacements parallel to the surface comes from interactions of the $\mathrm{DB}^{\prime} \mathrm{s}$ with the "back bonds" which are much
smaller than the first ones. This corresponding $2 \times 1$ superstructure 
tern is shown in Fig. 4.

It is interesting to note that recent low-energy electron diffraction (LEED) intensity calculations by Feder ${ }^{30}$, when compared with experimental LEED profiles seem to discriminate against the $\pi$-bonded chain model in favor of a buckling model.

However, we like to again stress the fact that the above phonon instabilities and the buckling displacement pattern are found for the ideal, unreconstructed $\mathrm{Si}(111)$ surface, and cannot predict a new equilibrium for the surface lattice structure. Our studies demonstrate how a microscopic phonon calculation can be carried our taking the actual surface

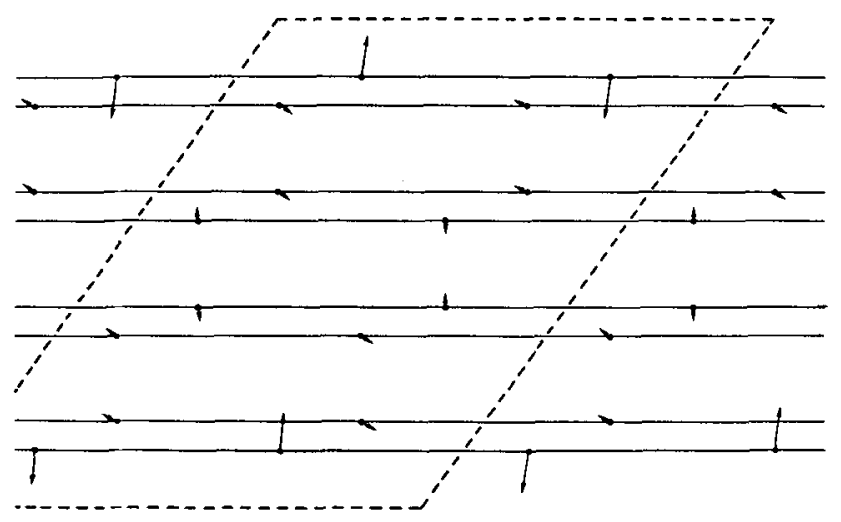

Fig. 4. Displacement pattern corresponding to the phonon instabilities at the $\mathrm{L}$ point. The dashed line shows the new unit cell for the $2 \times 1$ reconstruction surfaces. electronic density profile and inhomogeneities as well as many-electron effects carefully into account. Our interest was mainily to show, how one and the same concept of the Green's function description of (two-particle) elementary excitations can draw a coherent picture of the manybody physics of a given (for example a proposed reconstruction model) surface. Like in the bulk ${ }^{6}$ the next step has also to concentrate on one-electron (quasi-particle) excitations for the surface, calculated from a nonlocal and energy-de-

pendent self-energy and their comparison with photoemission. We strongly feel, that too much emphasis is presently placed on the direct comparison of local-density (LDA) electronic structure data with photoemission: it is well known that the LDA gives a quite accurate description of ground-state energies, however, the LDA eigenvalues of the Kohn-Sham equations are not directly to be taken as one-electron excitations ${ }^{30}$. In bulk semiconductors and insulators, the LDA results in up to $40_{0}^{\circ}$ too small band gaps. Presently, a study is undertaken, which expresses the non-local and energy-dependent self-energy for the electronic quasiparticles at a Si-surface in terms of the two-particle screening propagator. Thereby it is hoped to gain a similar quantitative description of surface one-electron excitations as in our previous bulk studies.

It is a pleasure to acknowledge fruitful discussions with Profs. A.A. Maradudin and W. Kohn.

\section{References:}

1. See for example, "Photoemission in Solids II" eds. L. Ley and M. Cardona, Topics in Applied Physics, Vol. 27 (Springer 1980). and Mönch W. Surf. Sci. 86 (1977) 682, and references therein.

2. Duke C.B., Paton A. and Ford W.K., Phys. Rev. B 24 (1981) 3310, and references therein. 
3. Ibach H. and Lewald S., J. Vac. Sci. Technol. 15 (1978) 407, and Raether H., Springer Tracts 38 (1965).

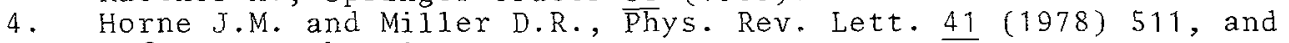
references therein.

5. For a review see for example, Hanke W., Adv. Phys. 27 (1978) 287 ; Hanke W. and Sham L.J., Phys. Rev. B 2 i (1980) 4656 .

6. Strinati G., Mattausch H.J. and Hanke W., Phys. Rev. Lett. $\underline{45}$ (1980) 290, Phys. Rev. B 25 (1982) 2867.

7. Hanke W., Mattausch H.J. and Strinati G., in "Electron-Correlations ed. by Devreese, J.T. (Plenum 1983).

8. Muramatsu A. and Hanke W., in "Ab-Initio Calculations of Phonon Spectra" ed. by Devreese J.T. (Plenum 1983).

9. Muramatsu A. and Hanke W., Phys. Rev. B 27 (1983) 2609

10. Muramatsu A. and Hanke W., Sol. St. Comm. 42 (1982) 537

11. Burstein E., Hartstein A., Schoenwald J., Maradudin A.A., Mills D.L. and Wallis R.F., in "Polaritons" eds. Burstein E. and de Martini F. (Pergamon, 1974). p. 89, and Wallis R.F., Progress in Surf. Science 4, ed. Davison S.G. (Pergamon, 1973), No. 3

12. For a recent review on surface waces see Maradudin A.A. in "Festkörperprobleme XXI" (1981), p. 25

13. Ludwig $W$. , in "Springer Tracts in Modern Physics" (Springer, 1967) Vol. 43; and Benedek G., Surf. Science 61 (1976) 603.

14. Interface phonons for the inversion layer Si(111) on $\mathrm{SiO}_{2}$ have been calculated by Kelly M.J. and Hanke W., Phys. Rev. B 23 (1981) 924

15. The possibility of superconductivity for inversion layer interfaces has been investigated by Kel1y M.J. and Hanke W., Phys. Rev. B 23 (1981) 112 .

16. Fedders P.A. and Martin P.C., Phys. Rev. 143 (1966) 245.

17. Chan S.K. and Heine V., J. Phys. F3 (1973) 795.

18. Halperin B.I. and Rice T.M., Sol. St. Phys. 21 (1968) 115.

19. Northrup J.E., Ihm J. and Cohen M.L., Phys. Rev. Lett. 47 (1981) 1910.

20. Himpsel F.J., Heimann P. and Eastman D.E., Phys. Rev. B 24 (1981) 2003.

21. Uhrberg R.I. G., Hansson G.V., Nicholls J.M. and Flodström S.A., Phys. Rev. Lett. 48 (1981) 1032.

22. Pandey K.C., Phys-Rev Lett. 47 (1981) 1913, and Phys. Rev. Lett. 49 (1982) 223.

23. Del Sole R. and Chadi D.J., Phys. Rev. B 24 (1981) 7431.

24. Allen R.E., Alldredge G.P. and deWette F. W., Phys. Rev. B4 (1971) 1661 .

25. Tong S.Y. and Maradudin A.A., Phys. Rev. 18 (1969) 1318

26. Zimmermann R., Appl. Phys. 3 (1974) 235.

27. Ludwig W., Japan J. App1. Phys. Suppl. 2 (1974) 879

28. Tosatti E. and Anderson P.W., Sol. St. Comm. 14 (1974) 713.

29. Chadi D.J., Surf. Sci. 99 (1980) 1.

30. Sham L.J. and Kohn W., Phys. Rev. 145 (1966) 561. 